\title{
NEWGARDEN: a computer program to model the population dynamics and genetics of establishing and fragmented plant populations
}

\author{
Stephan Pelikan $\cdot$ Steven H. Rogstad
}

Received: 21 July 2012/ Accepted: 22 January 2013/Published online: 3 February 2013

(c) The Author(s) 2013. This article is published with open access at Springerlink.com

\begin{abstract}
Conservation of plants often involves isolated in situ or ex situ protective restoration or supplemental plantings. Plant preservation also frequently involves maintenance of recently fragmented populations. The effects of the spatial patterning of the initial target species individuals in such situations on future population growth and genetic diversity maintenance are commonly difficult to predict, especially given that plants have varying life history characteristics. NEWGARDEN allows users to create a virtual preserve or fragment of specified size, place initial individuals at designated locations (the program is spatially explicit using a grid system), and follow population development stemming from bouts of mating influenced by user-specified life history characteristics (e.g., genetic diversity of the initial individuals; age-specific mortality; age-specific reproduction; gene dispersal distances; etc.). Output, which can be reported for the entire population data set and/or specified regions of the preserve or fragments after each bout of mating, includes total population and individual newest cohort data on the number of individuals, total number of founding alleles retained, observed heterozygosity, expected heterozygosity, and $\mathrm{F}_{\mathrm{IT}}$. Use of the program is illustrated with a NEWGARDEN analysis of the effects of varying founder pattern and gene dispersal distance on population growth and genetic diversity for an annual plant.
\end{abstract}

\section{S. Pelikan}

Department of Mathematical Sciences ML25, University

of Cincinnati, Cincinnati, OH 45221, USA

S. H. Rogstad ( $\square)$

Department of Biological Sciences ML6, University

of Cincinnati, Cincinnati, OH 45221, USA

e-mail: steven.rogstad@uc.edu
Keywords Conservation genetics - Founder effects · Fragmentation - Plant population genetics - Population establishment $\cdot$ Restoration genetics

\section{Introduction}

If current trends continue, conservation of numerous plant species will increasingly involve the creation and management of limited rescue or maintenance populations that will need to be manipulated in small, fragmented habitats. Several costs are associated with such management, and thus modeling population establishment, development, and consequent changes in genetic diversity under different conditions can assist in determining best practices for plant conservation efforts (e.g., Rogstad and Pelikan 2011).

NEWGARDEN is a computer program designed to model the growth and population genetics of plant populations developing from different founding and life history conditions. Although several other programs exist to simulate and analyze population characteristics and gene flow within, between, and among plant populations (e.g., see Balloux 2001; Machon et al. 2003; Guillot et al. 2005; Excoffier and Heckel 2006; Epperson et al. 2010; Eco-Gene and MetaPop (http://www.evoltree.eu/index.php/modelling-platform/models), VORTEX (http://www.vortex9.org/vortex.html), etc.), NEWGARDEN uses a unique suite of user-specifiable conditions for plant population modeling as outlined below. NEWGARDEN was created to provide a tool for researchers, students, and plant conservationists to explore how differing founding population characteristics as specified by the user will affect consequent population growth and diversity. Such comparative analyses can be used to assist in planning more effective plant introduction (in or ex situ) or population management strategies. The program is intended to be used 
to investigate small populations (usually $<50,000$ individuals) that are commonly of concern to conservation biologists.

NEWGARDEN populations do not develop based on theoretical modeling formulas, but rather from initial input stipulations that condition virtual matings and the establishment of offspring. A brief outline of the program-system requirements, input parameters, and output is given below.

\section{Program-system requirements}

The NEWGARDEN program is written in the Java computer language. It will run on any computer for which a current Java Virtual Machine is available. These can be downloaded at no cost for Windows, Mac, and Linux systems. Several versions are available. NEWGARDEN is tested using the JVM from Oracle available at http://java.com/en/download/ index.jsp.

\section{Input}

Trials One set of input conditions is referred to as a "trial."

Preserve or fragment description Populations develop on a grid system. Grid point density represents the average distance between two mature individuals at carrying capacity. The designated shape of the preserve-fragment system can include complex polygons and separated areas (in which case genes can flow between the areas, but individuals die immediately (see below) if dispersed into an intervening area), or areas connected by corridors (individuals can establish in the corridor).

Founding population The user designates the number of founders and places them at specified grid points. The founders are randomly selected from a virtual source population. The source population is an "ideal" population for which the user specifies the genetic diversity (number of loci to be analyzed, number of alleles at each locus, and the frequency of each allele). The level of inbreeding for the source population can be designated. The age and sex (cosexual, or microgamete-only or megagamete-only producing individuals) of each founder can be stipulated. Populations can thus include all cosexual or dioecious only individuals.

Population development The population develops through serial bouts of matings or "generations." The user inputs the number of generations for a given trial. Each new group of surviving offspring arising from a particular population mating is called a cohort. For non-annual species, individuals in each cohort increase in age together by one "year" with each new population bout of mating.

Mortality Individuals can die in several ways. Dead individuals are removed from any further processing immediately. Offspring die when: (1) an offspring is dispersed off the grid; (2) an offspring is dispersed to an already occupied grid point; or (3) it is dispersed to a grid point to which other offspring have been newly dispersed and is not chosen at random to be the sole survivor at that point. For non-annual species, individuals also die according to age-specific mortality rates specified by the user: individuals within age classes are randomly selected to die to meet the stipulated rate of mortality for that age class at each bout of mating. Annuals and short- or longlived perennials can be modeled by setting mortality rates appropriately.

Reproduction rate The number of offspring produced per individual of each age class (age-specific reproduction) during each bout of mating can be defined. We normally recommend that offspring production be distributed among eligible potential producers according to the Poisson distribution (Seed distribution method="poisson"), although other options exist (e.g., even distribution among eligible offspring producers; see Rogstad and Pelikan (2011) for more details). Offspring reproduction rate refers to the number of matings conducted regardless of their subsequent fate. For example, if a given mating results in the subsequent immediate death of the offspring due to dispersal off the preserve, or fails because no eligible pollinators are within range (see below), that attempt at successful mating is still counted as a mating event.

Pollen rate the relative contribution of pollen from each age class can be specified. For example, the user can stipulate that eligible (e.g., within distance: see below) pollen-donating individuals of age seven to 11 have a $5 \%$ chance of contributing pollen in a particular mating event compared to a $25 \%$ chance of pollen coming from individuals aged between 25 and 33. Pollen donation rates for all age classes are thus specified, and they must sum to $100 \%$.

Mating system The species can be designated as cosexual or dioecious. If cosexual, the species lacks a strong genetic self-incompatibility system as is the case with approximately 50 percent or more of angiosperms (Good-Avila and Stephenson 2002; Igic and Kohn 2006). However, if cosexual, the rate of selfing can be specified, ranging from no selfing, to selfing occurring at a rate equal to any other mating (true random mating), and up to $100 \%$.

Offspring dispersal The user can specify the proportion of offspring that are dispersed to different distance "frames" centered on the megagamete producing plant chosen for each mating even. If for a given offspring produced, the offspring is dispersed to a distance frame point outside of the grid system, the offspring dies immediately. 
Pollen dispersal The proportion of pollen contributed from different distance "frames" centered on the megagamete producing plant chosen for each mating can be stipulated. If no eligible pollen contributor occurs within the pollen frame selected for a mating, the mating counts as one mating event but fails to produce an offspring.

Replicate runs Each separate NEWGARDEN analysis of the input conditions for one trial is called a "run." The user can specify the number of runs, that is, replicate analyses, of the input conditions for one trial. Means and standard deviations across runs for a given trial are provided for relevant output statistics.

\section{Program output}

Summary statistics (mean and standard deviation) calculated across all the runs of a trial are provided each generation for both the total population and the most recently produced cohort. Output for the total population includes data from all existing individuals including all cohorts, while output termed "cohort" is taken only from the surviving individuals of the latest cohort produced in each round of mating. For annuals, the total population data and the cohort data should be exactly the same through generations, which will not be the case for non-annual species. Summary statistics are reported for population size, total number of founding alleles retained, observed and expected (Hardy-Weinberg equilibrium) heterozygosity, and $\mathrm{F}_{\mathrm{IT}}$. These statistics are produced for the total population and for subregions of the preserve/fragment as specified by the user.

The output is provided in comma separated variable (.csv) format allowing convenient import into most data management programs (Microsoft Excel, JMP, SYSTAT, $R$, etc.).

More detailed information concerning the input and output conventions discussed above can be found in Rogstad and Pelikan (2011).

\section{Comparative examples}

To demonstrate the use of NEWGARDEN, we created four sets of trial input (trials A through D) that were identical except as described below. The input files for these four trials are available at http://math.uc.edu/ pelikan/ NEWGARDEN.

\section{Conditions identical among trials}

Input conditions shared among these trials include (they are listed in the order they appear in the input file): the source population from which founders are randomly drawn has 20 loci, each of which has 100 alleles at equal frequencies of 0.01 (thus there are 2,000 alleles available in the source population); all individuals are cosexual; the average reproduction rate or potential offspring produced per individual $=1.15$ (we call this $\mathrm{r}$, but see Rogstad and Pelikan 2011); Poisson distribution of offspring produced across eligible individuals; all individuals have an equal likelihood of supplying pollen in a given mating subject to the distance constraints discussed below; the species is an annual (thus we report only total population output data below); selfing occurs at a rate equally probable to any other mating; the square preserve is 2,000 grid points on a side $\left(4 \times 10^{6}\right.$ points total; see Fig. 1$)$ and output is given only for this entire region; for each trial there are 100 replicate runs from which mean and standard deviation values are derived; there are 172 founders, each of which has two alleles for each of the 20 loci randomly drawn from the alleles available in the source population; the inbreeding coefficient $(\mathrm{F})$ for the source population $=0$.

Conditions that vary among trials

\section{Founder placement}

Trials $A$ and $C$ (Fig. 1) The 172 founders are placed in an approximate square of 13 lines with 13 individuals each (three lines had 14) with two grid points between individuals. The square is inset from the lower left corner borders of the preserve by 10 grid points from the $x$ and $y$ axes.

Trials $B$ and $D$ (Fig. 1) The 172 founders are placed in four lines of 43 individuals each with four grid points between individuals. The lines are positioned with the bottommost line running parallel to the preserve bottom $x$ axis, but raised above that $x$-axis by 21 grid points. The leftmost beginning individual of each line is inset from the preserve leftmost $y$-axis by 10 grid points.

\section{Dispersal distance}

Within all trials, pollen and offspring dispersal distance regimens are the same with pollen dispersal coming from or offspring distributed to four distance frames (in both the $\mathrm{x}$ and $\mathrm{y}$ directions) centered on the individual selected to produce the offspring in a particular mating. These distance frames, and probabilities of dispersal from or to each frame, varied among trials as follows.

Trials $A$ and $B$ Frame 1 runs from 0 (selfing or offspring deposition at the same point where the offspring producer existed) to 3 grid points; Frame 2 runs from 4 to 7 grid points; frame 3 runs from 8 to 11 grid points; frame 4 runs from 12 to 15 grid points. There is a $25 \%$ probability that 


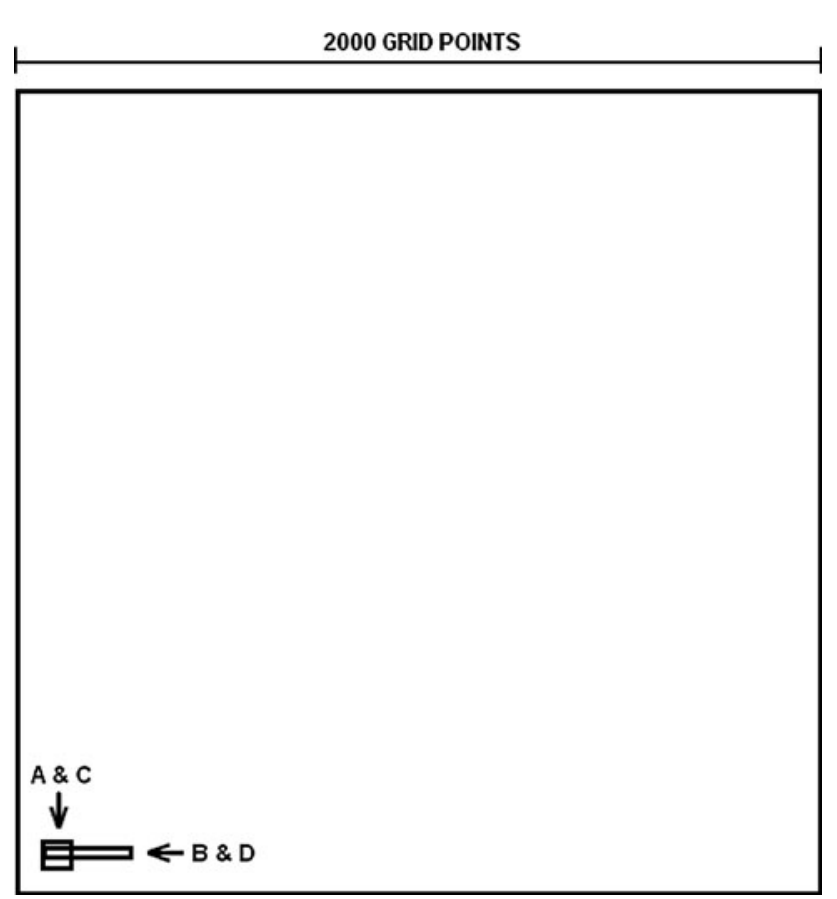

Fig. 1 Map of the square preserve (2,000 grid points on a side) showing approximate areas of placement of the 172 founders for each of the different trials. In trials $A$ and $C$, the founders are placed in a square of 13 lines, each with 13 individuals (three lines had 14), with two grid points between individuals. This square is inset from the preserve lower left corner by 10 grid points from the $x$ and $y$ axes. In trials $B$ and $D$, the founders are placed in 4 lines of 43 individuals each (found within the area indicated by the rectangle) with four grid points between individuals. The lines are parallel with the $x$ axis, the bottommost being raised 21 grid points above the $x$ axis. Each line is inset from the leftmost vertical preserve axis by 10 grid points. This figure is not to scale

pollen or offspring will come from or be distributed to any one of the frames. Altogether, dispersal range includes the closest 961 grid points. For these trials, dispersal will be said to be "short" in the following discussion.

Trials $\mathrm{C}$ and D: Frame 1 runs from 0 (selfing possible) to 7 grid points; Frame 2 runs from 8 to 15 grid points; frame 3 runs from 16 to 23 grid points; frame 4 runs from 24 to 31 grid points. Dispersal probabilities from or to frames: frame $1=40 \%$; frame $2=25 \%$; frame $3=25 \%$; frame $4=10 \%$. Altogether, dispersal range includes the closest 3,969 grid points. For these trials, dispersal will be said to be "distant."

\section{Results}

Under the given conditions, differences in positioning of founders and gene dispersal distances produce different mean population growth rates (Fig. 2), degrees of allele retention (Fig. 3), levels of observed heterozygosity
(Fig. 4), and $\mathrm{F}_{\mathrm{IT}}$ values (Fig. 5) as populations develop through generations. In the following discussion, mean values indicated as different have mean $t$ test $p$ values $\leq 0.05$.

After 14 bouts of mating with short dispersal, when less dense founders are placed in longer lines that are slightly more inset above the preserve bottom (trial B) compared to in a denser square nearer to the preserve bottom left corner (trial A), population growth increased by $76 \%$, allele retention increased by $17 \%$, and observed heterozygosity decreased by $5.1 \%$. If founders are placed in patterns similar to trial A versus trial $\mathrm{B}$, but dispersal is more distant (trials C vs. D, respectively), similar patterns of differences in population growth and mean founding alleles retained occur. Although trial D initially loses observed heterozygosity more rapidly than trial $\mathrm{C}$, the two have similar (although statistically different: $p$-value $=0.0041$ ) mean values at generation $14 . \mathrm{F}_{\mathrm{IT}}$ values are higher when founders are more inset into the preserve in less dense, longer lines compared to in less inset, denser squares whether dispersal is short or long (compare B versus A (short dispersal) and $\mathrm{D}$ versus $\mathrm{C}$ (long dispersal) in Fig. 5). Thus, $\mathrm{F}_{\mathrm{IT}}$

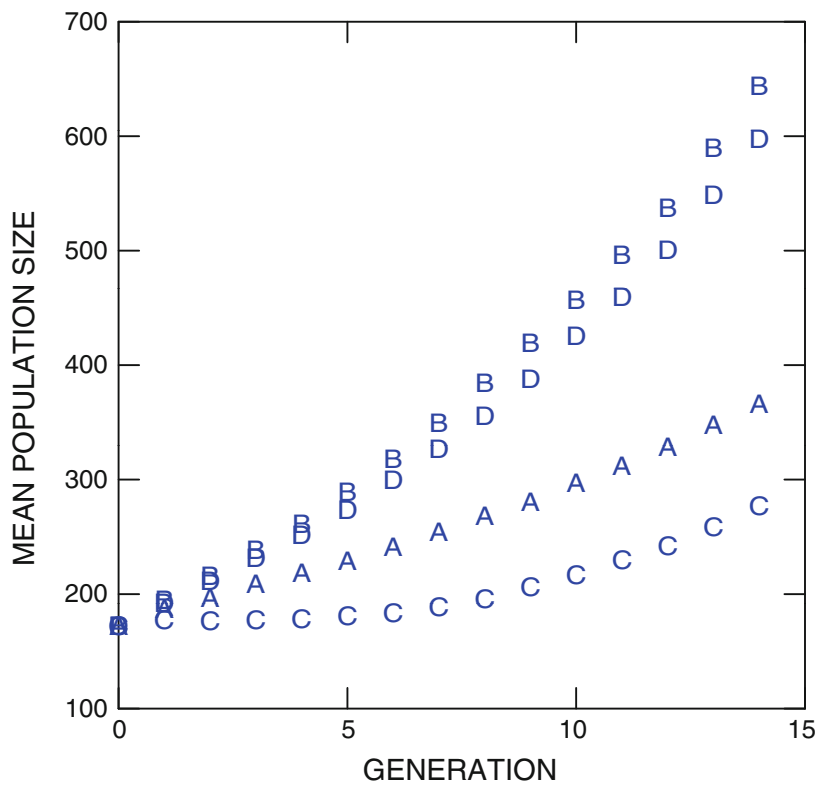

Fig. 2 Mean population size for four NEWGARDEN trials varying in founder geometry and gene dispersal distance. Conditions held identical across trials are given in the text. Populations differ in that trials $A$ and $C$ have the 172 founders in a square inset by 10 grid points from the lower left corner of the preserve and with two grid points between founders. Trials $B$ and $D$ have the 172 founders in four lines of 43 running parallel to the $x$-axis, with leftmost individuals inset from the $y$-axis by 10 grid points and the bottommost line inset above the lower border $x$-axis by 21 points; in trials $B$ and $D$ all founders separated by 4 grid points. Gene dispersal distance in trials $A$ and $B$ is "short", while it is "distant" for trials $C$ and D (see text for specifics of short vs. distant dispersal conditions) 


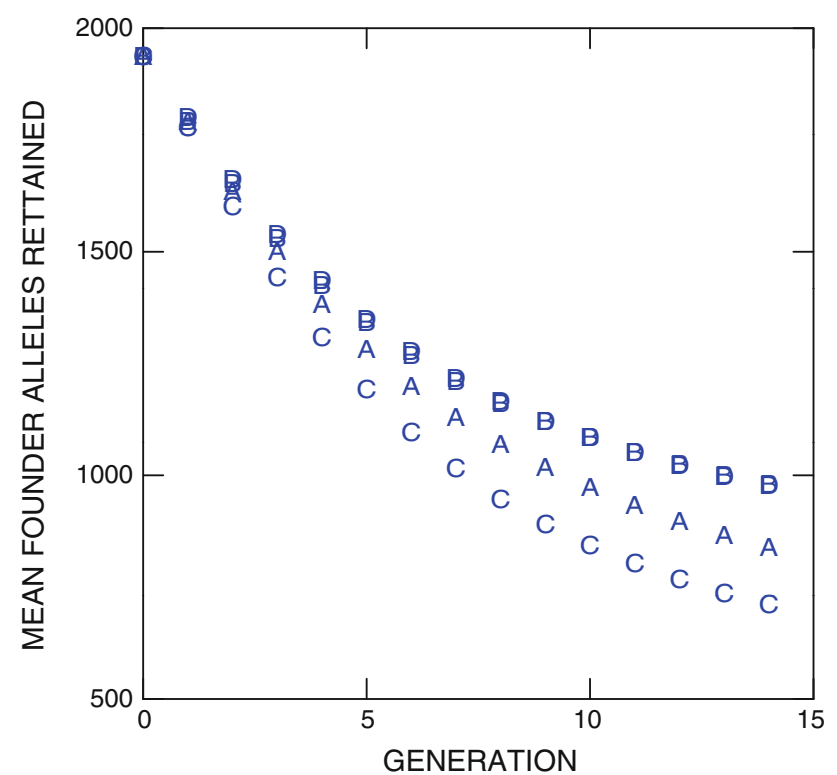

Fig. 3 The mean number of founding alleles retained in populations varying in founder geometry and gene dispersal distance. See the legends for Figs. 1 and 2, and the text, for details on how these trials differ

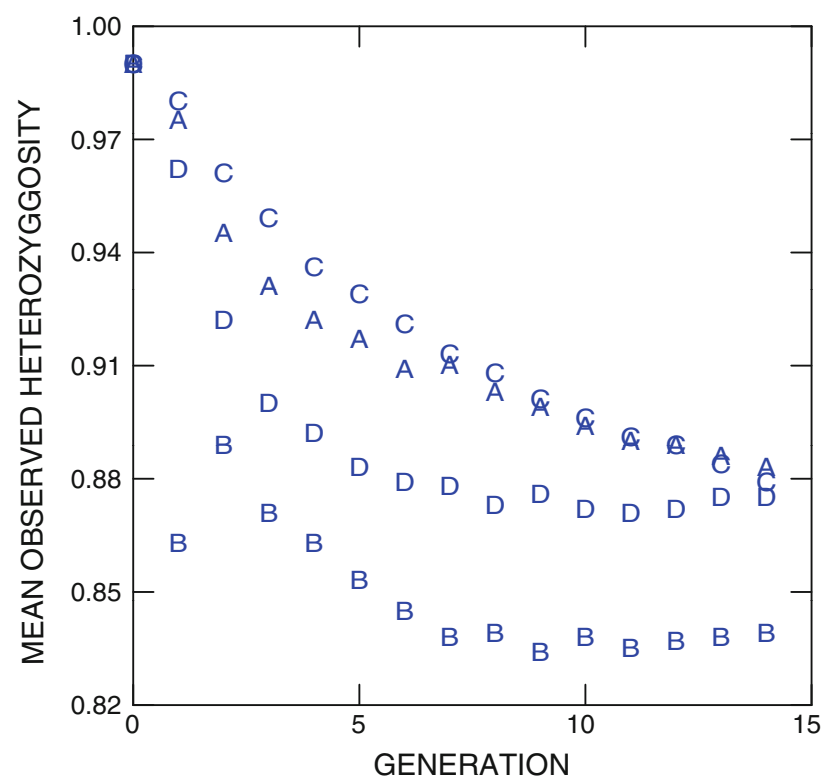

Fig. 4 The mean observed heterozygosity in populations varying in founder geometry and gene dispersal distance. See the legends for Figs. 1 and 2, and the text, for details on how these trials differ

increased when founders were more inset and positioned at greater distances in longer lines giving increased inbreeding and subdivision, although this effect was reduced when dispersal was long distance (contrast B vs. A compared to D vs. C).

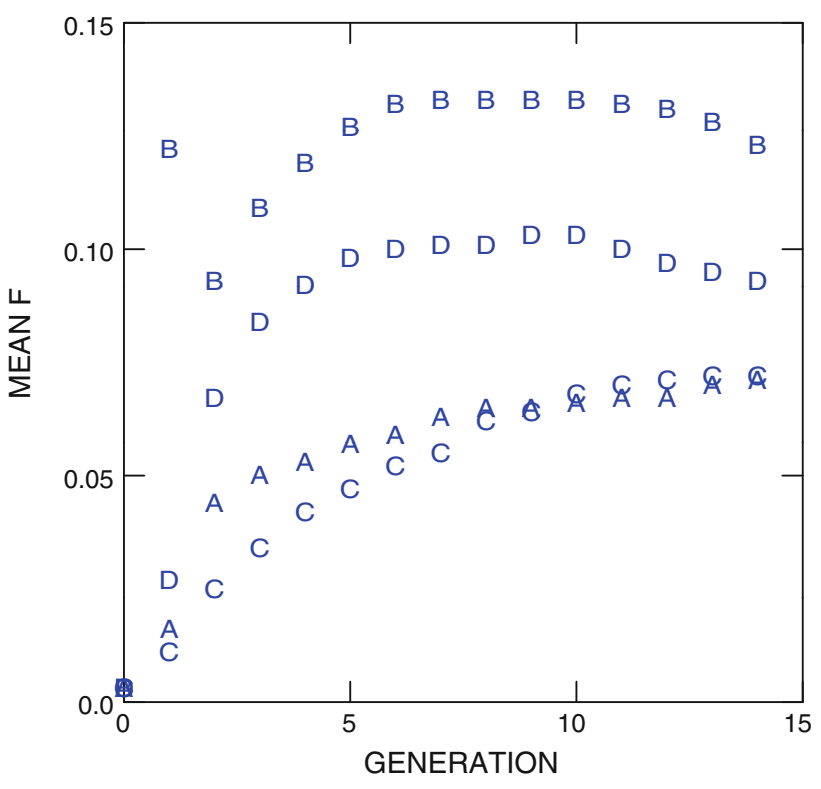

Fig. 5 Mean $F_{I T}$ in populations varying in founder geometry and gene dispersal distance. See the legends for Figs. 1 and 2, and the text, for details on how these trials differ

\section{Conclusions}

Plant conservation projects and evolutionary studies are often concerned with determining how founding conditions and life history characteristics interact to affect population growth, spread, and change in genetic diversity. Conservation practitioners in particular would benefit from modeling that could assist in selecting preservation practices that would be more likely to promote population growth and the retention of genetic variation for different plant species. The results above demonstrate that differing patterns of establishment combined with life history variation can produce a range of resulting population growth and genetic diversity characteristics. A more extensive examination of such establishment variation processes, mechanisms, and factors producing different outcomes, as well as some of the consequent biological and conservation implications, can be found in Rogstad and Pelikan (2011). NEWGARDEN can be used to explore how population establishment patterns and variation in life history characteristics can impact population growth and genetic diversity trajectories that can be set for generations to come. Obviously, multiple factors beyond those modeled with NEWGARDEN analyses can affect the establishment of any new population. Further, accurate information concerning the life-history characteristics used in NEWGARDEN modeling is not available for most plant species and populations, and the results presented here underscore the need to gather such information for target restoration 
species. However, the program can be used as an educational and research tool to explore how spatial and numerical variation in founder placement can affect population growth and genetics while remaining aware of such difficulties. By refining input to produce NEWGARDEN populations that grow in a similar fashion that reflects what is believed to have occurred with an existing stand, estimations of the previous and future growth and genetic diversity retention in that stand can also be made. Such estimations can be used to further model which types of manipulative interventions have the potential to be more effective in improving the growth and/or genetic diversity status of the stand going forward. Hopefully NEWGARDEN will contribute to improved understanding of some of the evolutionary processes at work in establishing plant populations, and to better informed conservation practices.

The program NEWGARDEN and associated materials, are freely available at http://math.uc.edu/ $\sim$ pelikan/NEW GARDEN. Future versions and information concerning the program will also be posted at that site.

Acknowledgments We thank A. Buck, C. Daley, M. Simkins, Y. Kashimshetty, and the Department of Biological Sciences and the Department of Mathematical Sciences, both at the University of Cincinnati.

Open Access This article is distributed under the terms of the Creative Commons Attribution License which permits any use, distribution, and reproduction in any medium, provided the original author(s) and the source are credited.

\section{References}

Balloux F (2001) EASYPOP (version 1.7): a computer program for population genetics simulations. J Hered 92:301-302

Epperson BK, McRae BH, Scribner K, Cushman SA, Rosenberg MS, Fortin MJ, James PMA, Murphy M, Manel S, Legendre P, Dale MRT (2010) Utility of computer simulations in landscape genetics. Mol Ecol 19:3549-3564

Excoffier L, Heckel G (2006) Computer programs for population genetics data analysis: a survival guide. Nat Rev 7:745

Good-Avila SV, Stephenson AG (2002) The inheritance of modifiers conferring self-fertility in the partially self-incompatible perennial Campanula rapunculoides L. (Campanulaceae). Evolution 56:263-272

Guillot G, Mortier F, Estoup A (2005) Geneland: a computer package for landscape genetics. Mol Ecol Notes 5:712-715

Igic B, Kohn JR (2006) Bias in the studies of outcrossing rate distributions. Evolution 60:1098-1103

Machon N, Bardin P, Mazer SJ, Moret J, Godelle B, Austerlitz F (2003) Relationship between genetic structure and seed and pollen dispersal in the endangered orchid Spiranthes spiralis. New Phytol 157:677-687

Rogstad SH, Pelikan S (2011) Genetic diversity in establishing plant populations. Science Publishers, Enfield 